\title{
PREDACIÓN DE Euseius emanus (EI Banhawy, 1979) SOBRE Oligonychus beeri Estebanes \& Baker, 1968
}

\author{
PREDATION OF Euseius emanus (EI Banhawy, 1979) ON \\ Oligonychus beeri Estebanes \& Baker, 1968
}

\author{
Lelia de Tomas de la Cruz ${ }^{1}$, Clorinda Vergara Cobián ${ }^{1,2}$, Alexander Rodríguez Berrío ${ }^{1}$, \\ Javier Huanca Maldonado ${ }^{3}$, Alfredo Giraldo Mendoza ${ }^{1}$ y Antonio Carlos Lofego ${ }^{4}$
}

\section{Resumen}

Se evaluó la capacidad de predación de protoninfas y adultos de E. emanus sobre huevos, larvas, protoninfas y adultos de $O$. beeri en condiciones controladas de temperatura y humedad. De acuerdo a los ensayos, las hembras adultas tuvieron mayor capacidad de predación (media $=23.18$ larvas) que las protoninfas (media $=11.15$ larvas) y los machos adultos (media $=8.05$ larvas). Ambos estados de desarrollo de E. emanus consumieron mayores cantidades de larvas y protoninfas, el consumo de adultos machos fue menor, mientras que el consumo de huevos fue mínimo.

Palabras clave: control biológico, capacidad de predación, Euseius emanus, Oligonychus beeri.

\begin{abstract}
The predation capacity of protonymphs and adults of E. emanus on eggs, larvae, protonymphs and adults of $O$. beeri was evaluated under controlled conditions of temperature and humidity. According to the tests, the adult females had a higher predation capacity (mean $=23.18$ larvae) than the protonymphs (mean $=11.15$ larvae) and the adult males (mean $=8.05$ larvae). Both stages of development of E. emanus consumed higher amounts of larvae and protonymphs, while their consumption of eggs was minimal.
\end{abstract}

Key words: biological control, predatory capacity, Euseius emanus, Oligonychus beeri.

\section{Introducción}

En ecosistemas agrícolas, los ácaros predadores, de la familia Phytoseiidae, son usados como agentes de control biológico de ácaros, trips y moscas blancas; existen experiencias de su papel en las regulaciones de ácaros fitófagos en frutales (Hardman et al., 1991; Walde et al., 1992; Solomon et al., 1993; Raudonis et al., 2004; Demite et al., 2014). En el Perú se tienen registros de especies de ácaros predadores en frutales (Guanilo, 2007; Muñoz, 2013; Muñoz \& Rodríguez, 2014). En el Perú, las especies de ácaros fitófagos del género Oligonychus Berlese, 1886, están reportadas como plagas en las zonas de producción agrícola (Gonzales \& Flechtmann, 1977).

En término de la preferencia alimenticia de la presa, la mayoría de los ácaros fitoseidos prefieren estadíos inmaduros, algunos huevecillos o adultos. En caso de algunas especies de arañitas rojas de los géneros Panonychus y Oligonychus, los huevecillos poseen una cáscara muy dura que impide una buena alimentación, limitando su predación sobre huevecillos (Pérez \& Marco, 2011).

Entre las especies predadoras importantes del género Euseius sobre ácaros fitófagos de la Familia Tetranychidae, se tiene a Euseius scutalis (AthiasHenriot, 1958) predador de Oligonychus perseae (Tuttle, Baker y Abbatiello, 1976) en huertos de palto en Israel (Maoz et al., 2010), y a
Euseius hibisci (Chant, 1959) predador de Oligonychus punicae (Hirst, 1926) en huertos de palto en California (McMurtry, 1985; Nomikou et al., 2001; Raza et al., 2005; Al-Shammer, 2010, citados por McMurtry et al., 2013).

El promedio diario de presas consumidas por una hembra Phytoseiidae es de 3 a 6 hembras, 10 a 20 larvas, ninfas y machos, y de 20 a 30 huevecillos de Tetranychidae (Badii et al., 2010; citando a Helle \& Sabelis, 1985)

Franco et al. (2008) citaron el potencial de predación de tres especies de fitoseidos: Iphiseiodes zuluagai Denmark \& Muma (1972), Euseius citrifolius Denmark \& Muma (1970) y Amblyseius herbicolus (Chant, 1959) sobre el ácaro del café Oligonychs ilicis (McGregor, 1917). E. citrifolius tiene preferencia por larvas y ninfas, siendo las hembras adultas las más eficientes, con $89.6 \%$ y $57.2 \%$ de predación (respectivamente), seguida de ninfas, que predan $55.6 \%$ de larvas y $22.8 \%$ de ninfas de $O$. ilicis.

Las fases jóvenes de E. citrifolius prefieren la fase larval de Tetranychus pacificus McGregor, aparentemente por la dificultad de perforar los huevos, siendo esto una explicación por la menor preferencia del estado de huevo (Moraes \& McMurtry, 1981; BlacKwood et al., 2004) citados por Cardoso et al. (2010). 
La capacidad de predación de los estados de desarrollo de Euseius alatus De Leon 1996: adulto macho, adulto hembra, larva y ninfa, sobre larvas de Oligonychus ilicis fue de 16.7, 20.3, 10.9 y 12.0 larvas predadas, respectivamente. Con respecto a la predación sobre ninfas de O. ilicis, fue de 13.7, 13.7, 8.0 y 5.8 ninfas predadas, respectivamente (De Toledo et al., 2013).

E. emanus es una especie nativa descrita de Perú (El-Banhawy, 1979) pero hasta la actualidad carece de información bibliográfica sobre aspectos biológicos y de comportamiento, por lo que el presente estudio fue determinar la capacidad de predación de protoninfas y adultos sobre huevos, larvas, protoninfas y adultos de $O$. beeri en condiciones de laboratorio.

\section{Materiales y Métodos}

El estudio se realizó en el Laboratorio de crianza de ácaros, ubicado en el Museo de Entomología Klaus Raven Büller (MEKRB) de la Universidad Nacional Agraria La Molina (UNALM), Lima / Perú, en condiciones controladas de temperatura $\left(25^{\circ} \mathrm{C}\right)$ y humedad relativa $(65-70 \%)$. El periodo de estudio fue de abril de 2018 a diciembre de 2019.

Los adultos de $O$. beeri se colectaron en hojas de palto (Persea americana) de una plantación ubicada dentro del campus de la UNALM. Los ácaros predadores, de la especie E. emanus, fueron obtenidos de la crianza masiva realizada en el laboratorio de crianza de ácaros MEKRB - UNALM. El material fue determinado a nivel de especies por los taxónomos en acarología del MEKRB y confirmados por el Dr. Antonio Lofego (UNESP) y el Dr. Ronald Ochoa (USDA), especialistas en Acarología.

Para la metodología de crianza del acaro predador y fitófago, se utilizó una adaptación de la metodología citada por Colcha (2013).

\section{Crianza de ácaros}

\section{ÁCARO FITÓFAGO, Oligonychus beeri}

La unidad de crianza fue un envase plástico rectangular de 1 litro de capacidad con tapa hermética y con una ventana superior cubierta con tela de organza, en la base interior se acondicionaron hojas maduras de palto sobre una esponja de $1 \mathrm{~cm}$ de altura cubierta con papel toalla y sumergida en agua destilada. En el borde de las hojas de palto se colocó papel toalla en tiras de $1.0 \mathrm{~cm}$ de ancho y el borde interno y superior del envase se untó con vaselina para impedir que los ácaros escapen de la unidad de crianza. El inicio de la crianza masal de los ácaros fitófagos fue a partir de huevos colocados diariamente durante tres días por 200 hembras silvestres por unidad de crianza.

Los estados de desarrollo huevo, larva, ninfa y adultos del ácaro fitófago, para los bioensayos, se obtuvieron a partir de huevos colocados por las hembras de la primera generación en las unidades de crianza con 10 o 15 parejas.

\section{ÁCARO PREDADOR, Euseius emanus}

La unidad de crianza fue un envase plástico rectangular de 1 litro de capacidad con tapa hermética y con una ventana superior cubierta con tela de organza, en la base interior se acondicionaron hojas de Acalipha wilkesiana Müll. Arg. (5.5 cm. x $7.5 \mathrm{~cm}$.) y el sustrato de oviposición utilizado fue lana negra de carnero, cubierta con $2 \mathrm{~cm}^{2}$ de láminas de polipropileno color negro.

En cada unidad de crianza se colocaron 20 machos y 20 hembras con la ayuda de un pincel $\mathrm{N}^{\circ} 000$; cada tres días se revisó el nivel de humedad y el aprovisionamiento de polen. Cada 15 días, como máximo, se realizó el cambio a nuevas unidades de crianza. Los ácaros predadores fueron alimentados cada tres días con polen de Ricinus communis L., 1753 y distintos estados de desarrollo de O. beeri. Las unidades de crianza con los ácaros se mantuvieron en un ambiente climatizado y controlado con $25{ }^{\circ} \mathrm{C}$ de temperatura, 65 a $70 \%$ HR y fotoperiodo $12: 12$, dentro de las instalaciones de investigación del MEKRB de la UNALM.

\section{Ensayos de predación}

Se usaron envases de plástico circulares con cierre hermético de 6 onzas de capacidad, acondicionados de forma similar a la crianza de mantenimiento: un agujero, una porción de hoja de palto sin infestar de aproximadamente $4 \mathrm{~cm}^{2}$ de área. En los bordes de las mismas se colocó papel toalla en tiras de $1 \mathrm{~cm}$ de ancho; en los bordes del envase, cerca de la esponja, se untó una línea de vaselina, ambos como barrera, para impedir la salida de la unidad de crianza de los estados de desarrollo móviles de la presa y de los estados de desarrollo de los ácaros predadores. Las unidades de predación se mantuvieron en ambiente con temperatura $25^{\circ} \mathrm{C}$ y $80 \% \mathrm{HR}$, con fotoperíodo de 12 horas luz, 12 horas oscuridad. Se realizaron evaluaciones de predación después de 24 horas para cada unidad.

Se evaluó la capacidad de predación de ninfas y adultos de E. emanus sobre cinco estados de desarrollo del ácaro fitófago: huevo, larva, ninfa (protoninfa), adulto macho y adulto hembra (Tabla 1). El ensayo testigo fue sin la presencia del ácaro predador.

Análisis de datos

Los resultados obtenidos en los ensayos de predación fueron analizados mediante la prueba estadística no paramétrica de Kruskall-Wallis. El análisis de capacidad de predación fue independiente para cada estado de desarrollo de la presa: huevos, larvas, protoninfas, adultos machos y adultos hembras.

\section{Resultados}

Los estados de desarrollo de E. emanus tuvieron, en general, una baja tasa de predación sobre los huevos de O. beeri. Las protoninfas, adultos machos y adultos hembras, consumieron una media de $0.15,0.15$ y 0.20 huevos, respectivamente, sin diferencias estadísticas entre estos valores (Tabla 2). La tasa de predación sobre 
las larvas de $O$. beeri tuvo valores significativamente distintos para cada estado de desarrollo de E. emanus. Los adultos hembras tuvieron la mayor tasa de predación con una media de 23.18 larvas, las protoninfas tuvieron una tasa de predación media de 11.15 larvas, y los adultos machos consumieron una media de 8.05 larvas (Tabla 2). La tasa de predación sobre las protoninfas de O. beeri tuvo valores significativamente distintos para cada estado de desarrollo de E. emanus. Los adultos hembras consumieron una media 13.40 protoninfas. Los adultos machos consumieron una media de 6.30 protoninfas y las protoninfas consumieron una media de 4.80 protoninfas (Tabla 2, Figura 1).

Tabla 1. Número de ensayos, tratamientos, variable respuesta, densidad de la presa y número de repeticiones por tratamiento.

\begin{tabular}{|c|c|c|c|c|}
\hline & 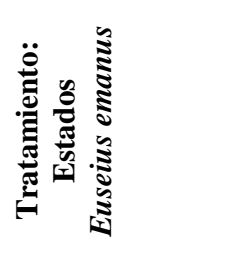 & 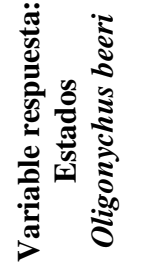 & 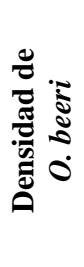 & 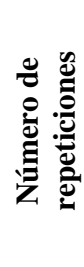 \\
\hline \multirow[t]{4}{*}{1} & Protoninfas & \multirow[t]{4}{*}{ Huevos } & 5 & 13 \\
\hline & Adultos machos & & 5 & 12 \\
\hline & Adultos hembras & & 5 & 16 \\
\hline & Testigo & & 5 & $*$ \\
\hline \multirow[t]{4}{*}{2} & Protoninfas & \multirow[t]{4}{*}{ Larvas } & 20 & 12 \\
\hline & Adultos machos & & 15 & 16 \\
\hline & Adultos hembras & & 33 & 22 \\
\hline & Testigo & & $*$ & $*$ \\
\hline \multirow[t]{4}{*}{3} & Protoninfas & \multirow[t]{4}{*}{ Protoninfas } & 10 & 10 \\
\hline & Adultos machos & & 20 & 10 \\
\hline & Adultos hembras & & 25 & 10 \\
\hline & Testigo & & $*$ & $*$ \\
\hline \multirow[t]{4}{*}{4} & Protoninfas & \multirow{4}{*}{$\begin{array}{l}\text { Adultos } \\
\text { machos }\end{array}$} & 5 & 10 \\
\hline & Adultos machos & & 10 & 16 \\
\hline & Adultos hembras & & 15 & 10 \\
\hline & Testigo & & $*$ & $*$ \\
\hline \multirow[t]{4}{*}{5} & Protoninfas & \multirow{4}{*}{$\begin{array}{l}\text { Adultos } \\
\text { hembras }\end{array}$} & 5 & 10 \\
\hline & Adultos machos & & 5 & 13 \\
\hline & Adultos hembras & & 5 & 15 \\
\hline & Testigo & & 5 & $*$ \\
\hline
\end{tabular}

Con respecto a la capacidad de predación de E. emanus sobre adultos machos de O. beeri, la mayor tasa de predación correspondió a los adultos machos con un consumo medio de 5.18 adultos machos; en tanto, los adultos hembras tuvieron un consumo medio de 3.90 adultos machos y las protoninfas tuvieron un consumo medio de 1.50 adultos machos. No se encontraron diferencias significativas en la predación sobre adultos machos (Tabla 2). La capacidad de predación de E. emanus sobre adultos hembras de $O$. beeri fue muy baja, encontrándose un consumo medio de $1.05,0.85$ y 0.40 , para adultos hembras, adultos machos y protoninfas respectivamente. No se determinaron diferencias significativas en la predación sobre adultos hembras (Tabla 2, Figura 1).

Tabla 2. Comparación de medias del consumo de huevos, larvas, protoninfas, adultos machos y adultos hembras de Oligonychus beeri por los diferentes estados de desarrollo de Euseius emanus.

\begin{tabular}{|c|c|c|c|}
\hline \multirow[b]{2}{*}{$\begin{array}{c}\text { Estados de } \\
\text { desarrollo de } \\
\text { O. beeri }\end{array}$} & \multicolumn{3}{|c|}{$\begin{array}{l}\text { Estados de desarrollo de } \\
\text { E. emanus }\end{array}$} \\
\hline & 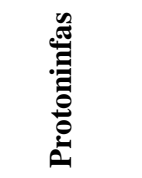 & $\begin{array}{l}\text { Adultos } \\
\text { machos }\end{array}$ & $\begin{array}{l}\text { Adultos } \\
\text { hembras }\end{array}$ \\
\hline Huevos & $0.15 \mathrm{a}$ & $0.15 \mathrm{a}$ & $0.20 \mathrm{a}$ \\
\hline Larvas & $11.15 \mathrm{~b}$ & $8.05 \mathrm{c}$ & $23.18 \mathrm{a}$ \\
\hline Protoninfas & $4.80 \mathrm{c}$ & $6.30 \mathrm{~b}$ & $13.40 \mathrm{a}$ \\
\hline Adultos machos & $1.50 \mathrm{~b}$ & $5.18 \mathrm{a}$ & $3.90 \mathrm{a}$ \\
\hline Adultos hembras & $0.40 \mathrm{a}$ & $0.85 \mathrm{a}$ & $1.05 \mathrm{a}$ \\
\hline
\end{tabular}

\section{Discusión}

La baja tasa de predación sobre huevos se puede explicar porque los huevos de las especies de los géneros Panonychus y Oligonychus tienen una cáscara muy dura que impedirían la alimentación por el ácaro predador. Así, Cardoso et al. (2010), en la actividad de predación de E. citrifolius sobre Tenuipalpus hevea Baker, 1945 mencionan que los estados inmaduros prefieren la fase larval de la presa, aparentemente por la dificultad de perforar los huevos; McMurtry et al. (2013) mencionan que los ácaros generalistas tienen como forma característica pequeños quelíceros con un diente pequeño y fijo en comparación con las especies de la subtribu Typhlodromalina, que presentan quelíceros de tamaño normal, con 6 a 12 dientes prominentes.

Los adultos hembra y macho de E. emanus mostraron un consumo promedio de 31.23 larvas de $O$. beeri, cercano al registrado por diversos autores en otras especies de ácaros predadores. Así, Rojas (2013) refiere 45 larvas de $O$. punicae por adulto de Amblyseius chunga Denmark y Muma, y 23.1 larvas de O. punicae por adulto de Euseius stipulatus (AthiasHenriot, 1960), en condiciones de laboratorio (22.8 \pm $1.6{ }^{\circ} \mathrm{C}$ y $\left.67 \% \pm 4 \mathrm{HR}\right)$. Así mismo, fue cercano a los resultados registrados por Franco et al. (2008) en el estudio del potencial de predación de tres especies de fitoseidos I. zuluagai, E. citrifolius y A. herbicolus, sobre $O$. ilicis, plaga del café, después de 24 horas. La hembra adulta de I. zuluagai tuvo un consumo promedio de 23.4 larvas, la de E. citrifolius, de 22.4 larvas y la de $A$. herbicolus, de 18.1 larvas de $O$. ilicis. De la misma forma, coinciden con los resultados obtenidos por De Toledo et al. (2013) en el estudio en condiciones de laboratorio para evaluar el potencial de predación de E. alatus sobre la hembra de O. ilicis sobre hojas de café; la hembra tuvo un consumo 
promedio de 20.3 larvas de O. ilicis. En estudios de predación de ácaros predadores de los géneros Amblyseius, Euseius y Typhlodromus sobre Frankliniella occidentalis (Pergande, 1895) concluyeron que la larva L1 fue más susceptible de ser predada, por su tamaño pequeño y menores recursos defensivos (Cardoso et al., 2010).

Estos resultados coinciden con lo encontrado por De Toledo et al. (2013), quienes observaron que la hembra de E. alatus fue más eficiente en la predación de las ninfas de $O$. ilicis, consumiendo 13.7 ninfas, y las ninfas de E. alatus tuvieron un consumo de 5.8 ninfas, cercanas al consumo promedio de las ninfas de E. emanus. Así mismo, coinciden con los estudios realizados por Franco et al. (2008), quienes determinaron un consumo promedio de 14.3 ninfas por las hembras de E. citrofolius, más que el consumo promedio de 9.12 ninfas por hembras de I. zuluagai y 7.2 ninfas por hembras de A. herbicolus. Del mismo modo, los resultados estuvieron cercanos a la predación de protoninfas de I. zuluagai al registrar un consumo promedio de 6.1 protoninfas de O. ilicis, ninfas de E. citrifolius con un consumo promedio de 5.7 protoninfas de $O$. ilicis.

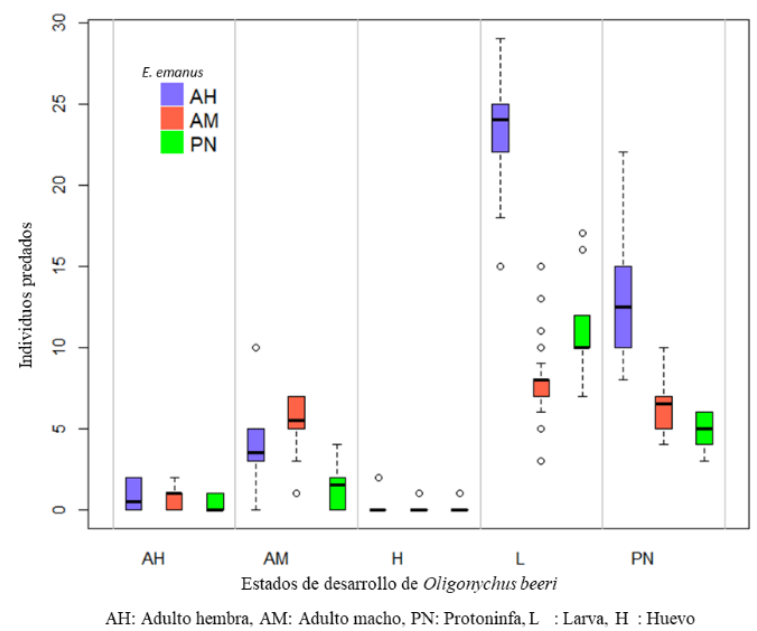

Figura 1. Predación de Euseius emanus (El Banhawy 1979) sobre Oligonychus beeri Estebanes \& Baker, 1968 , bajo condiciones de laboratorio a $25^{\circ} \mathrm{C}$ y $65-70 \%$ humedad relativa. La Molina, 2019.

La predación por adultos de E. emanus tiene resultados que se aproximan a los obtenidos por los adultos de I. zuluagai. El adulto hembra consumió en promedio 3.3 , el macho 1.1 y la ninfa 1.3 adultos de O. ilicis (Franco et al., 2008). No obstante, estos resultados, E. emanus tiene una mayor capacidad de predación que I. zuluagai. Los resultados son cercanos a los registrados en estudios de predación de adultos. Así, las ninfas y adultos machos de $I$. zuluagai tuvieron un consumo promedio de 1.3 y 1.1 adultos de $O$. ilicis, respectivamente; el consumo promedio de hembras de I. zuluagai, E. citrifolius y A. herbicolus fue de 3.3, 5.5 y 6 adultos, respectivamente (Franco et al., 2008). También, el consumo de 4.05 adultos machos y hembras de Oligonychus, por hembras de E. emanus, fue cercano al consumo promedio de 6 adultos de O. punicae por hembras de Phytoseiulus persimilis (Athias-Henriot, 1957) (García et al., 2017).

E. emanus es un ácaro predador de O. beeri clasificado en el estilo de vida Tipo IV, que se mantiene con dieta a base de presas de Oligonychus y polen. Esto último le confiere una participación sostenida, lo que le permite permanecer en los campos ante fluctuaciones bajas de la presa. En consecuencia, E. emanus es un ácaro predador potencial para ser utilizado en el Control Biológico de la arañita roja en programas de Manejo Integrado de Plagas.

\section{Conclusiones}

Los estados de desarrollo de Euseius emanus tuvieron una capacidad de predación efectiva sobre larvas, protoninfas y adultos de Oligonychus beeri en condiciones de laboratorio.

La predación de Euseius emanus fue mayor sobre larvas y protoninfas de Oligonychus beeri, siendo significativamente mayor para las hembras adultas.

\section{Agradecimientos}

Los autores expresan su agradecimiento al Programa Nacional de Investigación Agraria (PNIA), por el financiamiento del Proyecto 015/PNIA "Diversidad de ácaros predadores y su potencial uso como controladores biológicos en cultivos de palto y vid de la costa peruana". Asimismo, al Dr. Ronald Ochoa del USDA por su valioso apoyo en el proyecto.

\section{Literatura citada}

Badii M.H., Landeros J. \& Cerna E. 2010. Regulación poblacional de ácaros plaga de impacto agrícola. Daena: International Journal of Good Conscience, 5(1): 270-302. http://www.spentamexico.org/v5-n1/5(1)270-302.pdf.

Cardoso M.S.M., Vieira M.R., Figueira J.C. \& Silva H.A.S. 2010. Atividade predatória de Euseius citrifolius Denmark \& Muma (Acari: Phytoseiidae) sobre Tenuipalpus heveae Baker (Acari: Tenuipalpidae). Arq. Inst. Biol., São Paulo, 77(3): 471-476. http://www.biologico.sp.gov.br/uploads/docs/arq/v77_3/ cardoso.pdf.

Colcha R.S.E. 2013. Manual anual práctico de reproduccción masiva de Amblyseius californicus usado en el control biológico de Tetranychus urticae. Tesis Lic. UPS. Quito, Ecuador.

http://dspace.ups.edu.ec/handle/123456789/6049.

De Toledo M.A., Reis P.R., Da Silveira E.C., Marafeli P.P. \& De Souza P.G.C. 2013. Predadory potential of Euseius alatus (Phytoseiidae) on different life stages of Oligonychus ilicis (Tetranychidae) on coffee leaves under laboratory conditions. Neotrop. Entomol., 42(2): 185-190. DOI: https://doi.org/10.1007/s13744-0120100-6. 
Demite P.R., McMurtry J.A. \& De Moraes G.J. 2014 Phytoseiidae database: a website for taxonomic and distributional information on phytoseiid mites (Acari). Zootaxa, 3795(5): 571-577.

http://zoobank.org/urn:lsid:zoobank.org:pub:CAAFCFB 8-4864-41BE-89B0-8FE4CA3925B4.

El-Banhawy E.M. 1979. Records on phytoseiid (Acari) mites of Peru. International Journal of Acarology, 5(2): 111116. https://doi.org/10.1080/01647957908683133.

Franco R.A., Reis P.R., Zacarias M.S. \& Altoé B.F. 2008. Predation potential of three species of phytoseiids on Oligonychus ilicis (McGregor, 1917) (Acari: Tetranychidae). Coffee Science, 2(2): 175-182. http://www.coffeescience.ufla.br/index.php/Coffeescien ce/article/view/64.

García-Ángel O., Cerna-Chávez E., Aguirre-Uribe L.A., Ochoa-Fuentes Y.M., Chacón-Hernández J.C. \& Landeros-Flores J. 2017. Respuesta funcional de Phytoseiulus persimilis sobre Oligonychus punicae (Acari: Phytoseiidae, Tetranychidae) en hojas de aguacate. Acta Zoológica Mexicana (Nueva Serie), 33(3): 503-507.

DOI: https://doi.org/10.21829/azm.2017.3331151.

Gonzales R.H. \& Flechtmann C.H.W. 1977. Revisión de los ácaros fitófagos en el Perú y descripción de un nuevo género de Tetranychidae (Acari). Rev. Per. Ent., 20(1): 68-71.

https://www.revperuentomol.com.pe/index.php/revperu-entomol/article/view/654.

Guanilo A.D. 2007. Busca de Tetranychus evansis e seus inimigos naturais no Peru e no Norte da Argentina. Tesis Mag. Sci. USP Escola Superior de Agricultura "Luiz de Queiroz" Piracicaba. DOI: 10.11606/D.11.2008.tde11072008-143018.

Hardman J.M., Rogers R.E.L., Nyrop J.P. \& Frisch T. 1991 Effect of pesticide applications on abundance of European red mite (Acari: Tetranychidae) and Typhlodromus pyri (Acari: Phytoseiidae) in Nova Scotian apple orchads. Journal of Economic Entomology, 84(2): 570-580. DOI: https://doi.org/10.1093/jee/84.2.570.
Maoz Y., Gal S., Zilberstein M., Ishar Y., Alchanatis V., Coll M. \& Palevsky E. 2010. Determining an economic injury level for the persea mite, Oligonychus perseae, a new pest of avocado in Israel. Entomologia Experimentalis et Applicata, 138(2): 110-116.

DOI: https://doi.org/10.1111/j.1570-7458.2010.01080.x.

McMurtry J.A., De Moraes G.J. \& Sourassou N.F. 2013. Revision of the lifestyles of phytoseiid mites (Acari: Phytoseiidae) and implications for biological control strategies. Systematic \& Applied Acarology, 18(4): 297-320. DOI: http://dx.doi.org/10.11158/saa.18.4.1.

Muñoz J.L. 2013. Ácaros asociados al cultivo del palto (Persea americana Mill) en la costa central del Perú. Tesis Mag. Sci. Entomología. UNALM.

Muñoz J.L. \& Rodríguez A. 2014. Ácaros asociados al cultivo del aguacate (Persea americana Mill) en la costa central de Perú. Agronomía Costarricense, 38(1): 215-221. https://www.redalyc.org/pdf/436/43631007014.pdf. https://revistas.ucr.ac.cr/index.php/agrocost/article/view/ 15206.

Pérez-Moreno I. \& Marco-Mancebón V.S. 2011. Importancia y uso de los ácaros fitoseidos (Acari Phytoseiidae) en el manejo agroecológico de plagas. En: Aragón G.A., Jiménez-García D. \& Huerta L.M. (Eds.). Manejo Agroecológico de Sistemas (VOL. II). 69-92. Publicación especial de la Benemérita Universidad Autónoma de Puebla. Puebla, México.

Raudonis L., Surviliene E. \& Valiuskaite A. 2004. Toxicity of pesticides to predatory mites and insect in apple-tree site under field conditions. Environmental Toxicology, 19(4): 291-295. DOI: https://doi.org/10.1002/tox.20036.

Solomon M.G., Easterbrook M.A. \& Fitzgerald J.D. 1993. Mite-management programmes based on organophosphate-resistant Typhlodromus pyri in UK apple orchards. Crop Protection, 12(4): 249-254. DOI: https://doi.org/10.1016/0261-2194(93)90042-H.

Walde S.J., Nyrop J.P. \& Hardman J.M. 1992. Dynamics of Panonychus ulmi and Typhlodromus pyri: factors contributing to persitence. Experimental and Applied Acarology, 14: 261-291.

DOI: https://doi.org/10.1007/BF01200568.

\footnotetext{
${ }^{1}$ Universidad Nacional Agraria La Molina \Facultad de Agronomía \Departamento Académico de Entomología.

2 Correspondencia: cvc@lamolina.edu.pe.

${ }^{3}$ Universidad Nacional de San Agustín \Facultad de Ciencias Biológicas \Escuela Profesional de Biología.

${ }^{4}$ Universidade Estadual Paulista "Júlio de Mesquita Filho" (UNESP) \Departamento de Zoologia e Botânica.
} 